\title{
The influence of energetic surface heterogeneity on proton desorption during capillary filling of silica nanochannels
}

\author{
W. Piasecki · R. Charmas
}

Received: 31 October 2012/ Accepted: 25 January 2013/Published online: 9 February 2013

(c) The Author(s) 2013. This article is published with open access at Springerlink.com

\begin{abstract}
We applied the surface complexation model to describe the deprotonation of silica nanochannel walls as a function of $\mathrm{pH}$ and ionic strength of filling solution. We took into account energetic heterogeneity of silanol groups. We found that more heterogeneous walls of silica nanochannel will be more charged and can interact more strongly with ionic solutes. The modeling of nanochannels coated with 3-cyanopropydimethylchlorosilane is uncertain because charging mechanism of coated silica should take into account increased autolysis of interfacial water.
\end{abstract}

Keywords Silica - Nanochannels - Nanofluidics · Deprotonation · Energetic heterogeneity ·

Surface complexation

\section{Introduction}

A phenomenon where surface chemistry has a dominant influence on the properties of bulk fluid is the process of capillary filling of silicon dioxide nanochannels. In this case the key parameter is $\mathrm{pH}$ which has a dominant influence on electric charge of channel walls and the properties of molecules in the solution (Eijkel and Berg 2010; Napoli et al. 2010).

W. Piasecki $(\square)$

The Laboratory of Cosmetic Chemistry, The Faculty of Physical Education and Sport in Biała Podlaska, Józef Piłsudski University of Physical Education in Warsaw, Warsaw, Poland e-mail: piacho2@gazeta.pl

R. Charmas

The State Higher School of Computer Science and Business Administration in Łomża, Łomża, Poland
(Janssen et al. 2008) investigated the filling of silicon nanochannels with fluorescein solutions. They observed two zones in the fluid introducing into nanochannels. The front part of liquid was dark, and the back part exhibited fluorescence. The ratio of lengths of two zones was constant in time (Fig. 1a). The fluorescein molecule exhibits intense light emission when it is doubly deprotonated (for $\mathrm{pH}>7$ ), and it is inactive in protonated form (for $\mathrm{pH}<4$ ). It means that the $\mathrm{pH}$ in the dark zone is significantly lower than the $\mathrm{pH}$ in the bright zone. This increase in the concentration of hydrogen ions results from titration of solution by protons released from nanochannel walls (Fig. 2b).

The research on filling of silica nanochannels was continued by (Andersen et al. 2011) who analyzed the dependence of electrokinetic potential and the ratio of dark and fluorescent lengths on ionic strength for bare and 3-cyanopropydimethylchlorosilane coated silica nanochannels.

In this paper, we have developed the model describing surface deprotonation of silica nanochannels taking into account energetic heterogeneity of silanol groups. Next, we apply our model to describe experimental results published in literature so far. Finally, we give concise critical discussion of the modeling of coated silica nanochannels.

\section{Theory}

According to (Janssen et al. 2008) the balance of hydrogen ions in the investigated system can be calculated as follows.

The released number of protons in fluorescent zone is equal to:

$\mathrm{N}_{\mathrm{H}^{+}}^{\text {released }}=\mathrm{N}_{\mathrm{s}} \theta_{\text {dep }} \mathrm{L}_{\mathrm{FL}} 2(\mathrm{~h}+\mathrm{w})$

The number of protons absorbed by the solution in the dark zone is equal to: 


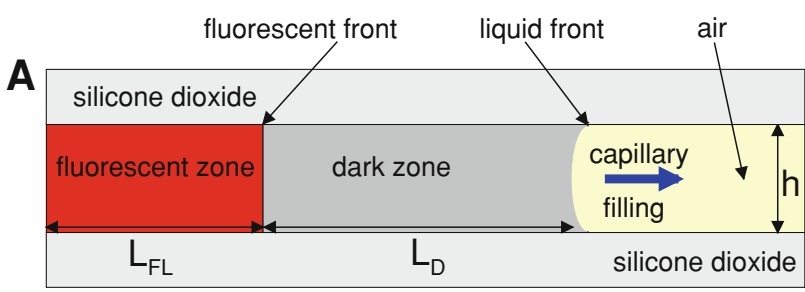

doubly charged fluorescein molecule (active form)

B

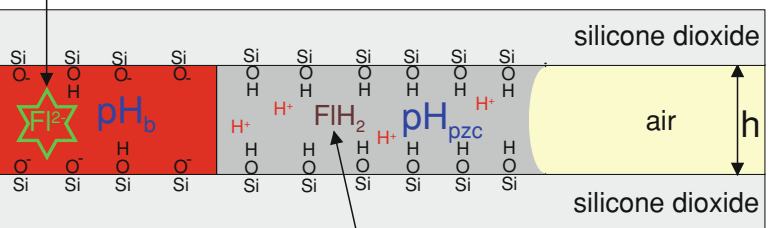

$\mathrm{pH}_{\mathrm{b}}>\mathrm{pH}_{\mathrm{pzc}} \quad \begin{aligned} & \text { uncharged fluorescein molecule } \\ & \text { (inactive form) }\end{aligned}$

Fig. 1 a The capillary filling of silicon dioxide nanochannel. Two zones in liquid can be distinguished (dark and fluorescent one). Ratio $\mathrm{L}_{\mathrm{D}} / \mathrm{L}_{\mathrm{FL}}$ is constant in time for given solution composition (ionic strength, buffer capacity). b The hydrogen ions released from nanochannel walls in the fluorescent zone move with filling solution to the dark zone where they decrease $\mathrm{pH}$ and quench fluorescein molecules by their protonation

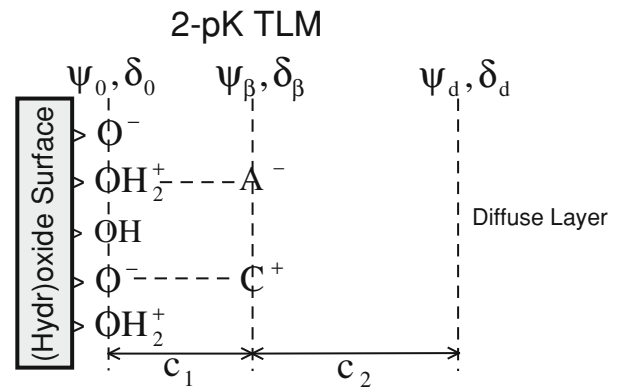

Fig. 2 The structure of the 2-pK triple layer model (2-pK TLM). Three planes can be distinguished: the surface plane $(0)$, the plane of adsorbed electrolyte ions $(\beta)$, and the plane being onset of diffuse layer (d). $\psi$ denotes electric potential and $\delta$ charge located in the individual planes. $c_{1}$ stands for inner-layer capacitance and $c_{2}$ is outer-layer capacitance

$N_{H^{+}}^{\text {absorbed }}=N_{A} B_{S} L_{D} h w$

The meaning of symbols in the above equations and in the Fig. 1 is following: $N_{A}$ is the Avogadro's number, $\mathrm{h}$ is the nanochannel height, $\mathrm{w}$ is the nanochannel width, $\mathrm{N}_{\mathrm{s}}$ is the number of silanol groups per $\mathrm{nm}^{2}, \theta_{\mathrm{dep}}$ is the fraction of deprotonated surface groups, $\mathrm{L}_{\mathrm{FL}}$ is the length of fluorescent zone, $\mathrm{L}_{\mathrm{D}}$ is the length of dark zone, $\mathrm{pH}_{\mathrm{B}}$ is the bulk $\mathrm{pH}$ of introduced solution, $\mathrm{pH}_{\mathrm{pzc}}$ is the $\mathrm{pH}$ at the point of zero charge of the silica surface, $\mathrm{B}_{\mathrm{S}}$ is the concentration of protons needed to change $\mathrm{pH}$ of solution from $\mathrm{pH}_{\mathrm{B}}$ to $\mathrm{pH}_{\mathrm{pzc}}$.
We can experimentally determine the number of protons released per area of nanochannel wall $\mathrm{N}_{\mathrm{H}^{+}}^{\text {exp }}$ assuming that $N_{H^{+}}^{\text {released }}=N_{H^{+}}^{\text {absorbed }}$ and applying $\mathrm{w}>>\mathrm{h}$ (because in rectangular nanochannel, its width is usually expressed in micrometers and its height in nanometers):

$N_{H^{+}}^{\exp }=N_{S} \theta_{\text {dep }}=N_{A} B_{S} h \frac{L_{D}}{2 L_{F L}}$

To analyze proton release in nanochannels we applied the 2-pK surface complexation model combined with the Triple Layer Model of interfacial region (2-pK TLM), which was depicted in Fig. 2 (Davis et al. 1978; Yates et al. 1974). The parameters of electric double layer in the 2-pK TLM are interconnected by the following expressions (Piasecki et al. 2001),

$\mathrm{c}_{1}=\frac{\delta_{0}}{\psi_{0}-\psi_{\beta}}$

$\mathrm{c}_{2}=\frac{-\delta_{\mathrm{d}}}{\psi_{\beta}-\psi_{\mathrm{d}}}$

$\psi_{\mathrm{d}}=\frac{2 \mathrm{kT}}{|\mathrm{z}| \mathrm{e}} \ln \left[\frac{-\delta_{\mathrm{d}}}{\sqrt{8 \varepsilon_{0} \varepsilon_{\mathrm{r}} \mathrm{kTI}}}+\sqrt{\frac{\delta_{\mathrm{d}}^{2}}{8 \varepsilon_{0} \varepsilon_{\mathrm{r}} \mathrm{kTI}}+1}\right]$

The equation (4c) follows from Gouy-Chapman theory of diffuse layer in electrolyte solutions.

The formation of surface complexes visible on Fig. 2 one can describe by the following reactions and equilibrium constants:

$\mathrm{SiO}^{-}+\mathrm{H}^{+} \leftrightarrow \mathrm{SiOH}^{0}, \mathrm{~K}_{0}$

$\mathrm{SiO}^{-}+2 \mathrm{H}^{+} \leftrightarrow \mathrm{SiOH}_{2}^{+}, \mathrm{K}_{+}$

$\mathrm{SiO}^{-}+\mathrm{C}^{+} \leftrightarrow \mathrm{SiO}^{-} \mathrm{C}^{+}, \mathrm{K}_{\mathrm{C}}$

$\mathrm{SiO}^{-}+2 \mathrm{H}^{+}+\mathrm{A}^{-} \leftrightarrow \mathrm{SiOH}_{2}^{+} \mathrm{A}^{-}, \mathrm{K}_{\mathrm{A}}$

where $\mathrm{SO}^{-}$denotes deprotonated silanol group at the oxide surface (empty adsorption site).

Additionally, in our model we have taken into account energetic heterogeneity of the silica surface. We have assumed that adsorption constants may have different values for different silanol groups. Assuming Gaussian-like distribution of adsorption energy (binding constants) and applying the the 2-pK TLM one arrives at the following expressions (heaving the form of Langmuir-Freundlich isotherms) for mean surface coverage by i-th adsorption complex (Rudzinski et al. 1999; Rudzinski and Everett 1992):

$\theta_{i t}=\frac{\left[\mathrm{K}_{\mathrm{i}} \mathrm{f}_{\mathrm{i}}\right]^{\mathrm{kT} / \mathrm{c}_{\mathrm{i}}}}{1+\sum_{\mathrm{i}}\left[\mathrm{K}_{\mathrm{i}} \mathrm{f}_{\mathrm{i}}\right]^{\mathrm{kT} / \mathrm{c}_{\mathrm{i}}}} \mathrm{i}=0,+, \mathrm{A}, \mathrm{C}$

where $\mathrm{kT} / \mathrm{c}_{\mathrm{i}}$ are the dimensionless heterogeneity parameters. To simplify the analysis, we assumed in our 
calculations that heterogeneity parameters of different complexes have the same value $\mathrm{kT} / \mathrm{c}_{\text {het }}$.

Next, $f_{i}$ can be expressed as functions of $\mathrm{pH}$ and electrolyte ions concentrations $\mathrm{a}_{\mathrm{C}}$ and $\mathrm{a}_{\mathrm{A}}$ :

$$
\begin{aligned}
& \mathrm{f}_{0}=\exp \left\{-\frac{\mathrm{e} \psi_{0}}{\mathrm{kT}}-2.3 \mathrm{pH}\right\} \\
& \mathrm{f}_{+}=\exp \left\{-\frac{2 \mathrm{e} \psi_{0}}{\mathrm{kT}}-4.6 \mathrm{pH}\right\} \\
& \mathrm{f}_{\mathrm{C}}=\mathrm{a}_{\mathrm{C}} \exp \left\{-\frac{\mathrm{e} \psi_{0}}{\mathrm{kT}}+\frac{\mathrm{e} \delta_{0}}{\mathrm{kTc} \mathrm{c}_{1}}\right\} \\
& \mathrm{f}_{\mathrm{A}}=\mathrm{a}_{\mathrm{A}} \exp \left\{-\frac{\mathrm{e} \psi_{0}}{\mathrm{kT}}-\frac{\mathrm{e} \delta_{0}}{\mathrm{kTc} c_{1}}-4.6 \mathrm{pH}\right\}
\end{aligned}
$$

The surface charge and diffuse layer charge can be expressed using surface coverages defined in eq (6),

$\delta_{0}=\mathrm{eN}_{\mathrm{s}}\left(\theta_{+\mathrm{t}}+\theta_{\mathrm{At}}-\theta_{\mathrm{Ct}}-\theta_{-\mathrm{t}}\right)$

$\delta_{\mathrm{d}}=\mathrm{eN}_{\mathrm{s}}\left(\theta_{-\mathrm{t}}-\theta_{+\mathrm{t}}\right)$

The total fraction of deprotonated surface groups can be calculated theoretically using the following equation,

$\mathrm{N}_{\mathrm{H}^{+}}^{\text {theory }}=\mathrm{N}_{\mathrm{s}} \theta_{\text {dep }}=\mathrm{N}_{\mathrm{s}}\left(\theta_{-\mathrm{t}}+\theta_{\mathrm{Ct}}-\theta_{+} \mathrm{t}-\theta_{\mathrm{At}}\right)$

The last value can be compared with experimentally determined number of protons released per area of nanochannel wall $\mathrm{N}_{\mathrm{H}^{+}}^{\exp }$, defined in eq (3).

To calculate the value of $\mathrm{N}_{\mathrm{H}^{+}}^{\text {thery }}$, first we have combined three equations $(4 \mathrm{a}-\mathrm{c})$ into one

$\psi_{0}-\frac{\delta_{0}}{\mathrm{c}_{1}}+\frac{\delta_{\mathrm{d}}}{\mathrm{c}_{2}}-\frac{2 \mathrm{kT}}{|\mathrm{z}| \mathrm{e}} \ln \left[\frac{-\delta_{\mathrm{d}}}{\sqrt{8 \varepsilon_{0} \varepsilon_{\mathrm{r}} \mathrm{kTI}}}+\sqrt{\frac{\delta_{\mathrm{d}}^{2}}{8 \varepsilon_{0} \varepsilon_{\mathrm{r}} \mathrm{kTI}}+1}\right]=0$

Next we have applied eqs $(8 b, 6,7)$ to express diffuse layer charge $\delta_{\mathrm{d}}$ as a function of $\delta_{0}$ and $\psi_{0}$. Finally, we have gotten two nonlinear equations (8a) and (10) with two unknowns $\delta_{0}$ and $\psi_{0}$. This system of equations can be solved numerically using mathematical software like Mathematica. Having determined the values of surface charge and potential for given $\mathrm{pH}$ and ionic strength we can easily calculate $\mathrm{N}_{\mathrm{H}^{+}}^{\text {theory }}$ from eq (9) or $\psi_{\mathrm{d}}$ from eq (4c).

\section{Results and Discussion}

In experiments performed by (Janssen et al. 2008) nanochannels were 40-50 nm deep and $20 \mu \mathrm{m}$ wide. They used two types of filling solutions: unbuffered $\mathrm{KCl}$ solution (mean $\mathrm{pH}=7.2$ ) and $\mathrm{KCl}$ solution buffered with TRIS (mean $\mathrm{pH}=8.5$ ). The both solutions contained fluorescein as pH-sensitive fluorescent dye. In Fig. 3, we have depicted

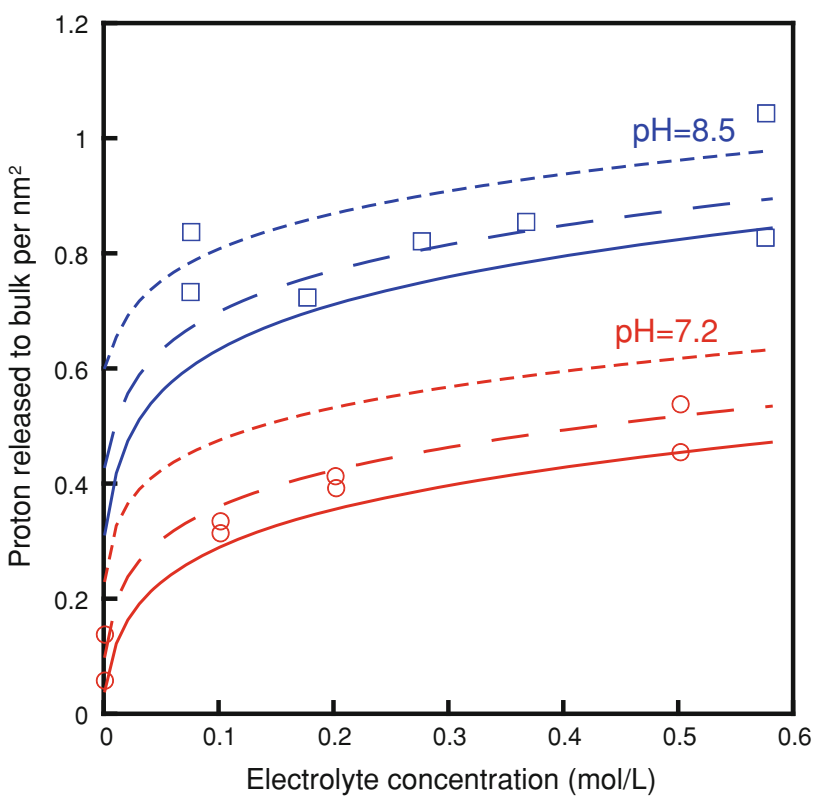

Fig. 3 The influence of energetic surface heterogeneity on the number of proton released to bulk per $\mathrm{nm}^{2}$ in silica nanochannel. Experimental data were measured for two different $\mathrm{pH}$ (i.e. $\mathrm{pH}_{\mathrm{B}}$ in fluorescent zone) and for various electrolyte concentration in filling solutions. The lines were plotted assuming various degrees of surface heterogeneity $\left(k T / c_{\text {het }}=1.0\right.$, homogenous surface, solid line; $k T / c_{\text {het }}=0.8$, dashed line, $k T / c_{\text {het }}=0.6$, finely dashed line $)$. The data were taken from (Janssen et al. 2008)

the number of protons released per area of nanochannel wall $\mathrm{N}_{\mathrm{H}^{+}}^{\exp }$ as a function of ionic strength.

Similar experiments were continued by (Andersen et al. 2011). However, these authors introduced some important modifications. First they determined electrokinetic potential in nanochannels using electro-osmotic current monitoring data, and second they modified nanochannel walls by coating silica surface with 3-cyanopropyldimethylchlorosilane. These authors used the solution of $\mathrm{KH}_{2} \mathrm{PO}_{4}$ as a filling buffer. In their experiments, nanochannel depth was in the range of 115-195 $\mathrm{nm}$ and their width changed in the range of 5-40 $\mu \mathrm{m}$. Buffer $\mathrm{pH}$ was equal to 7.2 (mean value). In Fig. 4, we presented results obtained by Andersen et al. There are data for bare silica surface and coated by cyanosilane.

Our aim was to fit the data for bare silica nanochannel obtained in two different laboratories using our model assuming surface energetic heterogeneity and only one parameter set. Because there is some controversy concerning charging mechanism of coated silica nanochannels we have not analyzed the data for modified nanochannels. This issue is discussed later in this paper.

We have fitted the data for bare nanochannels applying the parameters collected in Table 1 . The starting values of these parameters were taken from Sverjensky's paper 

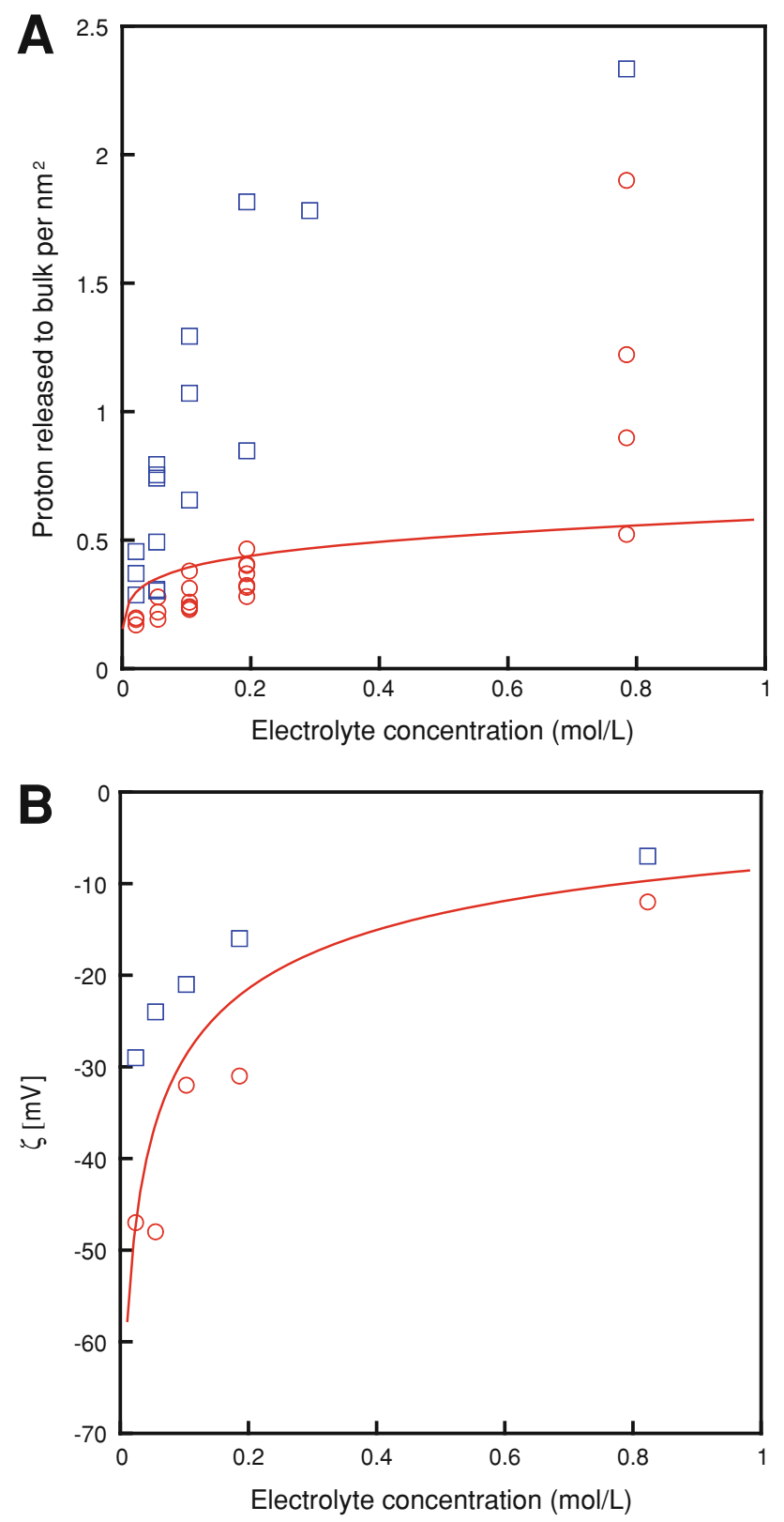

Fig. 4 The number of proton released to bulk per $\mathrm{nm}^{2}$ of wall (a) and electrokinetic potential (b) determined for bare (circles, red) and coated (squares, blue) silica nanochannels. Red line denotes the values calculated by using the parameters collected in the Table 1. For each ionic strength several measurements were done. The huge dispersion of data is visible for $\mathrm{I}=0.8$. The data were taken from (Andersen et al. 2011) (Color figure online)

(Sverjensky, 2005) for amorphous silica. This is quite recent, and the most reliable collection of parameters for the 2-pK TLM determined for different oxides and various electrolytes. However, these parameters can be treated as starting values, and their precise adjustment can be done.

Figure 3 shows how energetic surface heterogeneity affects on the number of proton released to bulk solution. The higher degree of surface heterogeneity means that the
Table 1 The parameter values used in the calculations

\begin{tabular}{ll}
\hline Parameter & Value \\
\hline$N_{S}$ & $\begin{array}{c}\text { 4.6 groups per } \\
\mathrm{nm}^{2}\end{array}$ \\
$\log K_{0}$ & 7.0 \\
$\log K_{+}$ & 5.6 \\
$\log K_{C}$ & -0.1 \\
$\log K_{A}$ & $\log K_{A}=\log K_{C}$ \\
$c_{1}$ & $1.2 \mathrm{~F} / \mathrm{m}^{2}$. \\
$c_{2}$ & $1.2 \mathrm{~F} / \mathrm{m}^{2}$. \\
$\frac{k T}{c_{\text {het }}}$ & 0.8 \\
\hline
\end{tabular}

surface is more deprotonated. We have found that the optimal value for heterogeneity parameter was $\mathrm{kT} / \mathrm{c}_{\text {het }}=0.8$.

To fit $\zeta$-potential data we had to increase outer Stern layer capacitance $c_{2}$ from standard value 0.2 to 1.2 . In our calculations we assumed that electric potential in d-plane $\psi_{\mathrm{d}}$ corresponded to measured electrokinetic $\zeta$-potential. The value of $c_{2}$ has very little impact on the degree of surface deprotonation, but significantly changes calculated $\zeta$-potential. Another way to fit $\zeta$-potential data is introducing the separation distance between outer Helmholtz plane (d-plane in Fig. 2) and slipping plane, where electrokinetic potential is measured (Kallay et al. 2012; Lutzenkirchen et al. 2008). This procedure requires an additional parameter (slip plane distance).

The crucial parameter in our modeling was the equilibrium constant for electrolyte cation adsorption $\mathrm{K}_{\mathrm{C}}$. We had to decrease its value from the initial one taking from Sverjensky's paper. The equilibrium constant for anion adsorption $\mathrm{K}_{\mathrm{A}}$ was insignificant in the modeling, because the investigated $\mathrm{pH}$ values were much higher than PZC of silica and anion adsorption was completely negligible.

Janssen et al. (2008) and Andersen et al. (2011) did also theoretical modeling of their own experimental data. The first group used the 2-pK Basic Stern Model neglecting electrolyte cation adsorption. The second group applied the 2-pK TLM including cation adsorption. The obtained results were satisfactory. In our opinion the modeling results for cyanosilane coated silica nanochannels are questionable.

According to Andersen et al. cyanosilane replaced about $25 \%$ silanol groups on the surface. Nevertheless coated silica exhibited much higher deprotonation than bare silica. Additionally, coated surface should have higher PZC value. Silanol groups should have different acidity (consequence of energetic heterogeneity), and the most acidic group should first react with cyanosilane molecules. This should increase PZC value of silica, and decrease the level of deprotonation. To fit the number of protons released to bulk solution Andersen et al. had to assume very high value 
for inner Stern layer capacitance $c_{1}\left(13-15 \mathrm{~F} / \mathrm{m}^{2}\right)$ which was about 20 times higher than the value used for bare silica surface.

In the simplest way capacitance $c_{1}$ can be defined by the following equation,

$\mathrm{c}_{1}=\frac{\varepsilon_{\mathrm{IL}}}{\mathrm{d}}$

where $\varepsilon_{\mathrm{IL}}$ is dielectric constant of the interfacial layer, and $\mathrm{d}$ is the distance between the silica surface and adsorbed electrolyte ions. It is not likely that any of these quantities could increase or decrease 20 times after surface coating.

One can try to explain this contradiction using the results from (Kallay et al. 2012; Lutzenkirchen et al. 2008). They found that for hydrophobic surfaces, there is an additional mechanism which increases the surface deprotonation. The silica surface which is coated by short hydrocarbon chains may enhance the autolysis of interfacial water molecules. As a result of this process, hydroxyl groups stay on the surface, and hydrogen ions are released to solution.

Andersen and co-workers assumed the following surface reaction with equilibrium constants $\mathrm{pK}_{+}^{\mathrm{A}}$ and $p K_{-}^{A}$,

$$
\begin{aligned}
& \mathrm{SiOH}_{2}^{+} \leftrightarrow \mathrm{SiOH}^{0}+\mathrm{H}^{+}, \mathrm{pK}_{+}^{\mathrm{A}} \\
& \mathrm{SiOH} \leftrightarrow \mathrm{SiO}^{-}+\mathrm{H}^{+}, \mathrm{pK}_{-}^{\mathrm{A}}
\end{aligned}
$$

In their calculations, they assumed that $\mathrm{pK}_{+}^{\mathrm{A}}$ is the key fitting parameter. The value of $\mathrm{pK}_{-}^{\mathrm{A}}$ was kept constant.

For $\mathrm{pH}>7$ the concentration of $\mathrm{SiOH}_{2}^{+}$groups on the silica surface should be very small, and in practice only the reaction $(12 \mathrm{~b})$ regulates the degree of surface deprotonation. Remembering that $\mathrm{PZC}=\frac{1}{2}\left(\mathrm{pK}_{+}^{\mathrm{A}}+\mathrm{pK}_{-}^{\mathrm{A}}\right)$ it is clear that the real key parameter was the PZC of the silica surface in the modeling described above. It is surprising that for fused silica, which covered the nanochannels, PZC value was not determined experimentally (it was assumed to be $\mathrm{PZC}=2.8$ ). It could be done, for example, by applying ISFET device or single crystal electrode technique (Kallay et al. 2007).

\section{Conclusions}

Surface complexation models like the 2-pK Triple Layer Model were successfully applied to describe the equilibria at oxide/electrolyte interface in silica nanochannels. However, for silica nanochannels coated by 3-cyanopropydimethylchlorosilane probably additional charging mechanism occurs. According to Kallay et al. the interfacial water on hydrophobic surfaces preferentially accumulates $\mathrm{OH}^{-}$ions, which increases hydrogen ions release to solution. This mechanism is not incorporated into surface complexation models. So, we have decided not to analyze the data for coated silica nanochannels.

Additionally, it has been shown that parameters values obtained by Andersen et al. who tried to fit the data for coated nanochannels were in conflict with the model assumptions.

For the bare silica nanochannels we have developed the 2-pK TLM assuming the surface energetic heterogeneity. Simply put, we assumed that different silanol groups should have various deprotonation constants. We have simultaneously analyzed the data obtained by Janssen et al. and by Andersen et al. We have used the parameter values predicted by Sverjensky for silica. We have found that more heterogeneous nanochannel walls were more charged. This means that the degree of surface energetic heterogeneity may influence on the transport of ionic solutes through nanochannels.

Acknowledgments The authors thank M.B. Andersen for providing extended experimental data from his paper.

Open Access This article is distributed under the terms of the Creative Commons Attribution License which permits any use, distribution, and reproduction in any medium, provided the original author(s) and the source are credited.

\section{References}

Andersen, M.B., Frey, J., Pennathur, S., Bruus, H.: Surface-dependent chemical equilibrium constants and capacitances for bare and 3-cyanopropyldimethylchlorosilane coated silica nanochannels. J. Colloid Interface Sci. 353, 301-310 (2011)

Davis, J.A., James, R.O., Leckie, J.O.: Surface ionization and complexation at the oxide/water interface I. Computation of electrical double layer properties in simple electrolytes. J. Colloid Interface Sci. 63, 480-499 (1978)

Eijkel, J.C.T., Berg, A.V.D.: Nanofluidics and the chemical potential applied to solvent and solute transport. Chem. Soc. Rev. 39, 957-973 (2010)

Janssen, K.G.H., Hoang, H.T., Floris, J., Vries, J.D., Tas, N.R., Eijkel, J.C.T., Hankemeier, T.: Solution titration by wall deprotonation during capillary filling of silicon oxide nanochannels. Anal. Chem. 80, 8095-8101 (2008)

Kallay, N., Preocanin, T., Supljika, F., Lutzenkirchen, J., Lovkovic, M.: Influence of interfacial water layer on surface properties of silver halides: effect of $\mathrm{pH}$ on the isoelectric point. J. Colloid Interface Sci. 375, 167-171 (2012)

Kallay, N., Preočanin, T., Ivšić, T.: Determination of surface potentials from the electrode potentials of a single-crystal electrode. J. Colloid Interface Sci. 309, 21-27 (2007)

Lutzenkirchen, J., Preocanin, T., Kallay, N.: A macroscopic water structure based model for describing charging phenomena at inert hydrophobic surfaces in aqueous electrolyte solutions. Phys. Chem. Chem. Phys. 10, 4946-4955 (2008)

Napoli, M., Pennathur, S., Eijkel, J.C.T.: Nanofluidic technology for biomolecule applications: a critical review. Lab Chip 10, 957-985 (2010)

Piasecki, W., Rudzinski, W., Charmas, R.: 1-pK and 2-pK protonation models in the theoretical description of simple ion adsorption at 
the oxide/electrolyte interface: a comparative study of the behavior of the surface charge, the individual isotherms of ions, and the accompanying electrokinetic effects. J. Phys. Chem. B. 105, 9755-9771 (2001)

Rudzinski, W., Charmas, R., Piasecki, W., Prelot, B., Thomas, F., Villieras, F., Cases, J.M.: Calorimetric effects of simple ion adsorption at the silica/electrolyte interface: quantitative analysis of surface heterogeneity. Langmuir 15, 5977-5983 (1999)
Rudzinski, W., Everett, D.H.: Adsorption of gases on heterogeneous surfaces. Academic Press, New York (1992)

Sverjensky, D.A.: Prediction of surface charge on oxides in salt solutions: revisions for 1:1 $\left(\mathrm{M}^{+} \mathrm{L}^{-}\right)$electrolytes. Geochimica et Cosmochimica Acta 69, 225-257 (2005)

Yates, D.E., Levine, S., Healy, T.W.: Site-binding model of the electrical double layer at the oxide/water interface. J. Chem. Soc. Faraday Trans. 70, 1807-1818 (1974) 